\title{
La función del funcionalismo: una exploración conceptual
}

HUGO GADENAS*

\section{Resumen}

El presente artículo tiene por objetivo analizar perspectivas actuales para una teoría funcionalista de la sociedad. En discusión con el funcionalismo de Talcott Parsons y con la propuesta sistémica de Niklas Luhmann, se propone un modelo de análisis funcional basado en el esquema problema/solución. Se discuten ventajas y limitaciones de esta propuesta y se proponen nuevos enfoques para el funcionalismo contemporáneo.

Palabras Clave: Teoría sociológica. Teoría antropológica. Funcionalismo. Teoría de sistemas sociales. Micro/macro.

* Universidad de Chile (Chile) 
Sociologias, Porto Alegre, ano 18, no 41, jan/abr 2016, p. 196-214

\section{The function of functionalism: a conceptual exploration}

\section{Abstract}

This paper aims to analyze current perspectives for a functionalist theory of society. Drawing on both the functionalist theory of Talcott Parsons and the systemic approach of Niklas Luhmann, a model for functional analysis based on the scheme problem/solution is proposed. The advantages and limitations of this proposal are then discussed, and new approaches to contemporary functionalism are proposed.

Keywords: Sociological theory. Anthropological theory. Functionalism. Social Systems Theory. Micro/macro.

\section{Introducción}

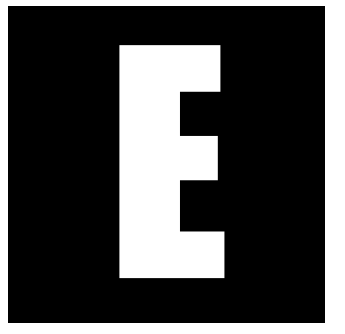

I título del presente artículo está planteado implícitamente entre signos de interrogación: ¿Cuál es la función del funcionalismo? La pregunta tiene al menos dos posibles respuestas. Por un lado, se puede reaccionar desde una teoría de la sociedad y atender a la función de la teoría funcionalista para la sociedad, sus aportes, limitaciones y también a sus intereses sociopolíticos, de clase, geopolíticos y de todo tipo. El mayor problema de este enfoque sería encontrar la estrategia más efectiva para que los datos den cuenta de estas relaciones entre teoría y sociedad, y su mayor virtud sería quizá mostrar que este tipo de análisis -tradicional o crítico- se atiene a una forma de argumentación típicamente funcionalista, vale decir, donde el funcionalismo cumple una función para algún actor, organización, ideología, etc.

Una segunda alternativa - y que en cierto modo engloba a la primera - es responder desde la teoría funcionalista en sí. Vale decir, prestar atención a los presupuestos del funcionalismo como teoría de la sociedad 
y su diseño conceptual. En este marco interpretativo, el problema principal es el concepto mismo de función y sus aspectos fundamentales. En lo sucesivo, este será el camino que escogeremos, pues representa un desafío tanto mayor a la primera opción, sobre la cual existe ya un debate establecido -aunque aún no se ha explorado la forma de argumentación subyacente a éste.

La estructura del artículo es la siguiente. En el primer apartado se presenta una breve introducción a la historia y los temas del pensamiento funcionalista, destacando la figura de Talcott Parsons como eje central. En el segundo apartado presentamos el cambio de paradigma funcionalista que propone Niklas Luhmann y sus implicancias teóricas. En el apartado tercero se incluyen propuestas tendientes a superar clásicos escollos del funcionalismo. En el aparado cuatro se analiza el funcionalismo a través de la distinción micro/macro y se exploran sus resultados. El artículo finaliza con un breve apartado de conclusiones sobre lo previamente expuesto.

\section{La razón funcionalista}

El funcionalismo pertenece a aquellas corrientes teóricas de las ciencias sociales de mayor antigüedad. Se puede incluso afirmar que es la más antigua de todas, si se considera a Auguste Comte (1875) como uno de sus padres fundadores - y a él mismo como un protofuncionalista. Lo cierto es que el pensamiento funcionalista está fuertemente anclado en la estructura profunda de las ciencias sociales y, a pesar de su permanente crisis de popularidad, difícilmente podrían darse por superados sus temas y conceptos.

Donde sea que se marque el inicio del pensamiento funcionalista en ciencias sociales, en la filosofía positiva de Auguste Comte o en los escritos de Émile Durkheim (2007 [1893]), es solamente con la antropología 
social británica de principios del siglo veinte que se estabiliza una corriente teórica distinguible como tal. Fueron las constantes pugnas por el reconocimiento de la autoría del concepto entre los antropólogos sociales Bronislaw Malinowski (1939; 1960) y Alfred R. Radcliffe-Brown (1952), lo que llamó la atención a las nacientes ciencias sociales de la época. Si bien el funcionalismo fue por décadas una denominación de origen de esta antropología social, influyó con fuerza el devenir de las ciencias sociales más allá de sus fronteras y fue especialmente en Estados Unidos donde alcanzó su mayor desarrollo. Su máximo exponente fue primero Robert K. Merton (1949) y luego su maestro Talcott Parsons. La popularidad de este funcionalismo si bien fue breve, su influencia fue lo suficientemente aplastante como para generar prontamente anticuerpos entre sus propias filas. Desde los años sesentas, fueron en gran parte los discípulos de Talcott Parsons quienes llevaron el estandarte en las sucesivas oleadas de la "revuelta" anti-funcionalista en Norteamérica (Alexander, 1987: 111 y ss.).

El motivo del descontento fue siempre el carácter totalizante del funcionalismo y debido a esto es que -no sin razón- hasta el día de hoy la palabra funcionalismo no goza de buena prensa (Nassehi, 2011: 58). Poco después de su primera gran obra teórica llamada La estructura de la acción social (1937), en la cual se abordaba una supuesta convergencia teórica espontánea entre las ideas de Alfred Marshall, Vilfredo Pareto, Émile Durkheim y Max Weber, Talcott Parsons reorientó su trabajo hacia una teoría funcionalista, debido en buena parte a su breve estadía en Londres, donde confiesa haber quedado profundamente impresionado con las lecciones de Malinowski (Parsons, 1970: 826) y con lo que él consideraba eran los intereses que compartía con este antropólogo (Parsons, 1960: 65). Con sus posteriores síntesis teóricas de 1951 - El sistema social y la obra colectiva Hacia una teoría de la acción social (Parsons, 1951; Parsons; Shils, 1951) - da el paso decisivo hacia una teoría funcionalista. 
Posterior a esa fecha, sus trabajos tendrán por propósito desarrollar este enfoque empleando un modelo conceptual sistémico-cibernético conocido como el AGIL (Parsons et al., 1953; Parsons; Smelser, 1956) y, en su obra tardía, conjugando este modelo con una teoría evolucionista (Parsons, $1964 ;$ 1966; 1971). Hacia el final de su carrera, Talcott Parsons era ya un sinónimo de funcionalismo, de su auge y su caída.

El descontento con el funcionalismo surgió con fuerza y de manera temprana, muy poco tiempo después de su ingreso al panteón de las teorías clásicas de las ciencias sociales. Parsons fue el motivo por el cual los sociólogos pragmáticos norteamericanos (especialmente los de Chicago) redescubrieron los escritos de Herbert Blumer (1937) - curiosamente contemporáneos a los primeros de Parsons -, en los cuales éste ungía al filósofo George Herbert Mead como padre de lo que denominaba "interaccionismo simbólico". Blumer aprovechó su momento de popularidad en la revuelta contra Parsons para reescribir sus antiguos ensayos (Blumer, 1969), esta vez lanzando dardos contra el funcionalismo parsoniano. Se reclutó a Erwin Goffman como un interaccionista, a pesar que éste se declaraba un funcionalista y simpatizante de la teoría parsoniana (Verhoeven, 1993), y Harold Garfinkel (1967: 68) formuló su propio enfoque - la etnometodología - renegando tempranamente de su maestro en Harvard y calificando con ironía al homo culturalis parsoniano como un simple "tonto" (dope) sin capacidad de juicio.

El funcionalismo era un ejemplo de teoría macro que volaba en un cielo demasiado alto y debía ser llevada al mundano terreno de lo micro, de la vida cotidiana. El estructuralismo de Claude Lévi-Strauss (1995) en Francia era el blanco de la misma crítica, así como el marxismo ortodoxo en Alemania mediante la "crítica" - inspirada parcialmente en Max Weber - de Max Horkheimer y Theodor W. Adorno (1998), también redescubierta hacia fines de los años 1960 aunque su formulación inicial es de 1944. 
El resto es historia conocida. El funcionalismo dejó de ser una práctica atractiva en los ámbitos académicos y se pusieron en tela de juicio la mayoría de sus pretensiones explicativas. Sólo un puñado de parsonianos sobrevivió a las revueltas (entre ellos Jeffrey C. Alexander, Paul Colomy, Richard Münch y José Almaraz) y los que en parte se valieron de sus planteamientos, tuvieron que replantear gran parte del legado parsoniano. Así ocurrió, por ejemplo, en la conocida disputa entre Niklas Luhmann y Jürgen Habermas (1971) que tuvo como trasfondo precisamente el funcionalismo parsoniano, el cual para Luhmann debía ser completamente reformulado y para Habermas debía ser aceptado en su forma ortodoxa sólo de manera parcial, es decir, sólo al nivel de los sistemas políticos y económicos que, a fin de cuentas, eran los únicos "sistemas" - aunque con signo negativo - para la teoría crítica de Adorno que inspiraba entonces a Habermas.

El cultivo - y en ocasiones también el culto - del funcionalismo ha quedado prácticamente sin adherentes, e intentos como el de Alexander y Colomy de fundar un "neofuncionalismo" (1992) no han ido más allá de las buenas intenciones. El panorama no es alentador cuando se pregunta entonces, ¿qué hacer?

\section{Sistema y función}

En cierta medida la crítica al funcionalismo tiene que ver con su imagen de la sociedad. En todas sus versiones, la teoría funcionalista adopta un modelo organicista de sociedad, donde el concepto de función sirve para explicar las relaciones entre un todo (organismo) y sus partes diferenciadas (órganos). Dicha relación se define en términos de "necesidad", es el todo el que precisa que sus partes satisfagan determinadas necesidades mediante tareas diferenciadas. Es así como diferencia la función 
de la utilidad de un hecho social Durkheim (2001: 140 y ss.) y el método funcional debe establecer este mismo tipo de relaciones en el análisis de Malinowski $(1939,1960)$ y de Radcliffe-Brown (1952), variando entre estos últimos únicamente el alcance de la relación establecida.

Talcott Parsons, ayudado de la teoría de sistemas, llevó este tipo de análisis a un nivel de sofisticación mucho mayor. Su primer modelo de 1951 definía tres sistemas (cultural, social y personalidad) con sus respectivas necesidades, y en sus ensayos posteriores perfecciona este modelo añadiendo un sistema orgánico como base material y energética para los demás sistemas. Su teoría de la evolución (Parsons, 1964; 1966; 1971), así como sus ensayos sobre los procesos que ocurren entre estos sistemas, es decir, sobre los medios simbólicos generalizados (Parsons, 1963ä; 1963b; 1968), tienen como base a la sociedad como un sistema que satisface sus necesidades vitales mediante subsistemas diferenciados.

La sociedad es, como resultado, un sistema que se mantendrá estable en tanto se satisfagan sus necesidades. La evolución de la sociedad no lleva a la inestabilidad sino más bien a resolver paulatina y diferenciadamente los problemas derivados del AGIL, es decir, del aumento de la "adaptación", "diferenciación", "inclusión" y "generalización de valores" (Parsons, 1966), como respuestas estructuralmente adecuadas, y a incorporar selectivamente en el desarrollo histórico de cada sociedad ciertos "universales evolutivos" (Parsons, 1964).

Como hemos visto, este tipo de teorización de un carácter más abstracto, general y estructural, fue la fuente de mayores cuestionamientos al funcionalismo parsoniano. Previo a la publicación de las síntesis teóricas funcionalistas de Parsons, Robert K. Merton ya había llamado la atención acerca de las dificultades de una empresa funcionalista como la que iba a emprender su maestro de Harvard. Clamaba entonces Merton (1949) - en un sentido claramente microsociológico - por teorías de alcance medio, 
por identificar funciones latentes a nivel empírico y por observar de manera más cercana los motivos de los agentes para orientarse de acuerdo o en desacuerdo con las normas socialmente aceptadas ${ }^{1}$. Lo que desató Parsons luego, pudo ser para Merton, siguiendo su propia terminología, una "profecía autocumplida". Los embates más duros contra el funcionalismo vinieron de teorías microsociológicas orientadas hacia prácticas cotidianas, hacia las interacciones y el conocimiento de la vida cotidiana. Desde entonces, esta crítica al funcionalismo se ha vuelto un lugar común.

No sin dificultades ha podido desarrollarse la teoría de sistemas sociales posterior a Parsons, intentando liberarse de esta pesada carga de determinismo. La propuesta de Niklas Luhmann (1970) por reformular la teoría funcionalista cuenta entre las estrategias mejor diseñadas para romper el rígido esquema funcionalista de necesidades/satisfactores.

Se trata en primer término de cuestionar el "realismo analítico" de Parsons (1937). La teoría de los - tres y luego cuatro - sistemas de Parsons propone un sistema de acción que debe responder a un entorno que presiona por cambios en el sistema. Frente a esto, Luhmann (1997) redefine la teoría de sistemas usando recursos constructivistas. La sociedad se diferencia de su entorno en términos de complejidad, ésta es menos compleja que su entorno y responde a éste con mecanismos de reducción. Complejidad es entonces una variable del sistema y no del entorno, por tanto los problemas se producen en el sistema y no se importan desde afuera.

Siguiendo este mismo razonamiento, la función es un esquema de observación construido por un observador que distingue causas y efectos (necesidad/respuesta, por ejemplo) y no se trata de una propiedad intrínseca a un sistema estable. "La función no es ningún efecto a producir, sino un esquema de sentido regulador, el cual organiza un área de comparaci-

\footnotetext{
${ }^{1}$ Margaret Archer (1996: 2 y ss.) coincide bastante con estas advertencias tempranas al funcionalismo, sin citar directamente a Merton.
} 
ón para rendimientos equivalentes" (Luhmann, 1970: 14). Esta propuesta de un funcionalismo de equivalencias implica que las relaciones causales deterministas son solamente una alternativa entre otras: "La función no es considerada como una especie peculiar de relación causal, sino que a la inversa, la causalidad es vista como un caso especial de aplicación de categorías funcionales" (Luhmann, 1970: 10).

Este cambio de paradigma implica no solamente mover el concepto de función hacia una teoría de la observación, sino también replantear la teoría de sistemas sociales que está en su base. No se trata de sistemas estables, cuya viabilidad depende de satisfacer necesidades determinadas, sino de sistemas inestables que operan en el sentido, es decir, que en cada una de sus operaciones producen su propia unidad sistema/entorno y deben enfrentar un horizonte contingente y abierto. La función no es, así, una presión de selección del entorno, sino una manera de manejar la complejidad interna al sistema, una manera entre otras.

De acuerdo con Luhmann (1970), la función es un esquema de observación del mundo bajo la forma problema/solución y ambos lados permanecen abiertos y contingentes. El funcionalismo clásico decidió estabilizar solamente un lado de esta forma, definió a los problemas (necesidades) como el lado estable y a las soluciones (satisfactores) como el lado variable, y los debates entre funcionalistas se alimentaron entonces del disenso relativo al inventario y nivel de las necesidades. Así, Malinowski $(1939 ; 1960)$ identificó una jerarquía de necesidades², desde lo bio-psicológico hasta lo simbólico; Radcliffe-Brown (1952) prefirió restringirse solamente a necesidades sociales de carácter orgánico, siguiendo

\footnotetext{
${ }^{2}$ Jerarquía incompleta si se considera que existen dos versiones del catálogo de necesidades. En sus escritos publicados póstumamente desaparece la necesidad de "descanso" y sus concomitantes satisfactores institucionales y se la reemplaza por la necesidad de "salud" y sus instituciones de "higiene" (cf. Malinowski, 1960: 91 y ss.).
} 
declaradamente a Durkheim, y Parsons (1951; Parsons; Smelser, 1956) finalmente estipuló primero tres y luego cuatro necesidades fundamentales a todo sistema de acción. Estos modelos funcionan de manera coherente solamente hasta el punto en que dicho modelo se hace reflexivo, y se abre allí la pregunta por la función de las funciones, es decir, por la necesidad que cubre la determinación de una necesidad.

Luhmann no escapa totalmente de este determinismo, aunque fundamenta de mejor manera sus elecciones teóricas. Es decir, la función de un sistema es resultado de su evolución y no es un a priori estructural. A nivel de la teoría de la sociedad, cada subsistema funcional se caracteriza por la codificación de comunicaciones en torno a problemas sociales autodefinidos y de relevancia generalizada, ésta es su función señala Luhmann (1997). La política democrática, por ejemplo, codifica al mundo como gobierno u oposición, y especifica un problema propio, esto es, producir decisiones colectivamente vinculantes. La contingencia de la política radica en que este problema puede ser una alternativa de acción para un actor cualquiera, quien puede preferir no politizar sus opciones, pero la función del sistema político es irremplazable. Se puede renunciar a solucionar un problema políticamente, pero al hacerlo se deben buscar alternativas funcionales, las que operan con otros códigos y poseen otras funciones. El riesgo que implica asumir una alternativa nueva asegura así la vigencia del sistema.

Si se deja la discusión en este punto, no se ven salidas claras a los típicos cuestionamientos al funcionalismo. Lo que ha ofrecido Luhmann como funcionalismo de equivalencias se plasma posteriormente, no obstante, en una teoría de la sociedad moderna que en algunos aspectos recuerda mucho a las listas de necesidades y satisfactores del funcionalismo clásico, que este autor pretende superar. Así, cada sistema funcional se encarga de resolver un problema específico, posee una codificación 
y programación propia, e incluso medios simbólicos, en un estilo muy similar a las matrices de Malinowski y del mismo Parsons. En este sentido, la teoría funcionalista quedaría relegada por opción propia a su territorio conocido y protegida solamente por su aislamiento.

3. La praxis funcionalista

El concepto de sistema social llevado a sus diferentes planos hace posible mirar al funcionalismo desde una óptica diferente. No se trata de capitular ante la microsociología y redescubrir que se pueden observar funciones y disfunciones, manifiestas o latentes, en la vida cotidiana o de perfeccionar diferentes facetas del AGIL (Münch, 1982), sino de testear el método funcional en diferentes escenarios y bajo diversas presiones. Basándose en el esquema de planos o niveles de sistemas sociales de Luhmann (1975), el cual distingue solamente tres niveles: interacción, organización y sociedad, podemos añadir como sistemas intermedios a grupos, redes u otros tipos de sistemas sociales ${ }^{3}$.

Armin Nassehi (2011: 62 y ss.) aconseja, en este sentido, un "funcionalismo operativo", es decir, focalizar la atención en el plano de las operaciones, de las prácticas y del(los) presente(s). Su estrategia consiste en mantener el esquema problema/solución, pero desplazar la mirada hacia la solución, es decir, preguntar: "para qué problema puede ser tal o cual práctica una solución" (Nassehi, 2011: 65). Esto lleva a Nassehi a defender una postura metodológica de carácter hermenéutico y etnometodológico. Esta maniobra resulta instructiva respecto de las alternativas que el método funcional posibilita, pero remite solamente a la crítica microsociológica.

El método funcional se puede conducir de manera más radical, es decir, probarse como explicación científica en la praxis de observación de la sociedad, en todos sus niveles. Para esto, no es suficiente con cambiar

\footnotetext{
${ }^{3}$ No me es posible extenderme en este punto acá. El argumento que sostengo es que entre sociedad, organización e interacción, se presentan un conjunto de sistemas sociales que no se ajustan a esta triada conceptual. (Cf. Cadenas, 2014)
} 
el foco hacia las soluciones, sino que se hace preciso subrayar el carácter contingente del esquema problema/solución en ambos lados, y ver cómo éste se comporta en diferentes niveles.

En primer término, la forma problema/solución prueba ser solución a un problema autoconstruido en sistemas de comunicación codificada, i.e. sistemas funcionales, los que a su vez producen nuevos problemas con cada solución. A nivel de estos sistemas puede operar el esquema funcional de manera reflexiva y, con esto, generalizarse a nivel de la sociedad. Con cada problema científico se construyen soluciones funcionalmente equivalentes y estas, a su vez, pueden referir a problemas igualmente equivalentes y crear otros nuevos, para el propio sistema o para otros sistemas. La penicilina probó ser solución ante el problema autoconstruido de la proliferación de los microbios, los cuales, como señala Bruno Latour (1993), surgieron únicamente gracias a la praxis científica. Los métodos de Pasteur se probaron exitosamente luego como solución técnica para la limpieza de la leche, y gracias a esto posteriormente el problema de su consumo generalizado como política pública.

Las interacciones pueden tener una reflexividad similar para el tratamiento del esquema problema/solución, pero su generalización es más bien limitada. Cada tratamiento del esquema problema/solución puede tratarse con cualquiera de los dos lados, i.e. puede ser solución o problema. Como aconseja Nassehi, puede preguntarse qué problema(s) se resuelve(n) con tal o cual práctica (solución), por ejemplo, con acostumbrar regalar flores a la pareja en alguna festividad o qué problemas se crean con dicha solución y las posibles soluciones equivalentes. A diferencia de los sistemas funcionales, en las interacciones puede perderse de vista en cualquier momento la práctica de solucionar problemas, problematizar soluciones o preguntarse por el esquema problema/solución (como 
puede suceder en un seminario sobre funcionalismo), en tanto se mantenga como horizonte una comunicación con sentido.

Entre interacciones y sociedad las limitaciones son más rígidas, a pesar que el esquema funcional posea mayor relevancia. Las organizaciones conocen pocos equivalentes funcionales a sus problemas autodefinidos y tienen que echar mano a rendimientos extraorganizacionales (otros miembros, otras prestaciones) cuando fracasan sus esquemas. La práctica burocrática pública o privada puede, bien "ritualizarse" repitiendo las mismas soluciones a diferentes problemas, bien asumirse como una organización que se especializa en asignar problemas y soluciones de la manera más "eficiente" posible, o quizás pueden agobiarse sus procedimientos ante problemas que no conocen soluciones. En cualquier caso, resulta siempre una tarea difícil el cambio de estas prácticas en las organizaciones.

Grupos, redes sociales, u otros sistemas sociales intermedios, presentan situaciones similares a organizaciones e interacciones. Debido a su naturaleza dual, presencial o ausencial, puede ser relevante para ellos no solamente un uso "técnico", es decir simplificado, del esquema de manera rígida o flexible, o simplemente no afrontarlo en su historia.

Lo que se quiere resaltar con esto, es que si bien se puede llevar el método funcional con éxito a diferentes niveles de construcción sistémica, la forma de observación, el esquema problema/solución, adquiere una relevancia central solamente a nivel de sistemas funcionales, a pesar que interacciones, organizaciones y sistemas intermedios también operan con sus propios problemas y soluciones. Si se trata de analizar la constante construcción de problemas, se puede concordar con Nassehi respecto de la relevancia que adquieren los sistemas funcionales y que al invertir el foco de atención, resulta más adecuada una postura más bien pragmática o etnometodológica, un "funcionalismo operativo". 


\section{Entre lo micro y lo macro}

En la discusión relativa a los rendimientos micro o macro del funcionalismo no puede darse por cerrado el debate aduciendo que éste presenta mayores rendimientos a nivel micro -como parece sostener Nassehi- o que habría que prestar mayor atención a lo macro -que pudiese ser una crítica al enfoque adoptado hasta ahora. La distinción micro/macro esconde un problema que debe ser, al menos, mencionado. Antes de esto, cabe sintetizar brevemente el punto de vista escogido.

Entiendo a los sistemas sociales como limitaciones, vale decir operaciones, construidas en una praxis comunicativa autopoiética, las cuales fijan momentáneamente restricciones a la contingencia del mundo mediante marcas de sentido, sociales, factuales y temporales. Un sistema social surge cuando hay por lo menos dos conciencias que vivencian o actúan y se toman en cuenta para sus posteriores acciones o vivencias, y al hacerlo limitan al menos momentáneamente su libertad -consciente o inconscientemente, la distinción es por ahora irrelevante- en un mundo que de pronto se encuentra habitado por un otro conocido o desconocido. No requieren de estar presentes y percibirse en un tiempo y espacio simultáneo, sino de experimentar esta indeterminación en las conciencias, sin poder anticipar el curso de las acciones por la sola e individual voluntad de una de las partes.

En este sentido, no se trata de sistemas de acción -o de acción comunicativa- determinados por las intenciones de un agente. En lugar de eso, el agente construye expectativas. Que en cada conciencia se pueda simular y ensayar la comunicación sin sufrir mayores consecuencias, apoya la catálisis de formación de sistemas sociales, pero no se puede construir el sistema sin al menos contar con un horizonte de negaciones contingentes e imposibles de anticipar empíricamente. En un mundo desolado, una conciencia solitaria dará prontamente con el hecho que el 
soliloquio sólo puede ser comunicación que no es comunicación, esto es, que el hablante tendrá que vivenciarse como un conocido desconocido, como un oyente que nunca antes ha escuchado lo que acaba de escuchar $y$, a riesgo de que dicha paradoja lo haga desaparecer de su propio mundo con sentido, hacer del tiempo una corriente sin memoria ni continuidad.

La estructura del sistema social, es decir, las expectativas que encuentran conexión con otras expectativas en un momento del tiempo, son marcas que condicionan la motivación para participar de la comunicación. Es decir, invitan o desalientan a asumir riesgos. Intervenir en una interacción sin invitación o presentación previa, hacerse pasar por estudiante de una institución sin haberse inscrito o intentar convertir la economía monetaria en una de trueque, son todas apuestas riesgosas, no imposibles. El "peso" del sistema está puesto tanto en la expectativa de éxito como de fracaso.

Con esto sigo muy de cerca los planteamientos de Luhmann (1997), pero es mi intención llevar estos más allá de la práctica teórica luhmanniana más ortodoxa. No solamente hay más entre sociedad e interacción que simplemente organizaciones, como he señalado algo apresuradamente, sino que el método funcional no requiere de justificaciones o restricciones micro- o macrosociológicas.

Contra la distinción micro/macro, según la cual los sistemas funcionales serían el polo de lo macro y las interacciones lo micro, puede argumentarse que los sistemas funcionales no son solamente macro-sistemas, es decir, sistemas que impactan a gran escala y que poseen estructuras complejas, programas, o semánticas históricas diferenciadas, sino que son también sistemas "pequeños", mínimamente codificados. En tanto unidades operativas, los sistemas funcionales codifican sus opciones de manera binaria, en valores positivos y negativos. Es así como un pago es el sistema económico, ya sea una transacción de activos en la bolsa de valores o la compra de cigarrillos en el almacén del barrio. Lo relevante es pagar en 
un horizonte de no pagos posibles. Por el contrario, las interacciones son también sistemas "grandes", en tanto deben procesar una gran cantidad de información generada en la co-presencia de los participantes, en un tiempo breve y respetando secuencias y turnos. En este sentido, la complejidad de las interacciones no puede ser despreciada.

Para evitar esta confusión, y en caso de querer mantener la distinción micro/macro, ésta puede basarse en un criterio de inclusión y exclusión. De este modo, los macro-sistemas serían aquellos que incluyen a nivel social de manera generalizada, mientras que los microsistemas serían los sistemas que requieren inclusiones mínimas y que operan de manera situacional e "indexical" (Garfinkel, 1967). Un pago es accesible a cualquiera que pueda pagar, así como cualquiera puede demandar un derecho, comunicar la trascendencia del alma o practicar un deporte. La interacción, en cambio, precisa de presencias específicas, de manejo de contextos y de retroalimentación. Basta con desconocer a los participantes, no manejar su idioma o su simpatía, para estar excluido.

El método funcional es la forma de operación de los sistemas funcionales, hemos dicho anteriormente, y a pesar de esto, el esquema problema/ solución es una forma generalizada de codificación del mundo, por tanto accesible a interacciones, organizaciones y sistemas intermedios. Ahora bien, la forma problema/solución puede ser aplicada como esquema de observación científica a cualquier sistema social, en tanto se defina este esquema como problema científico a resolver, o como solución a diversos e indeterminados problemas. Lo relevante es mantener abiertas las opciones de la forma en ambos lados para la observación empírica de sistemas sociales.

\section{Conclusiones}

Difícilmente pueda con esto mejorarse la reputación del funcionalismo o motivar una revisión más profunda de sus ideas y controversias. Una 
popularidad tan repentina puede no ser recomendable para una teoría que aún no cicatriza sus heridas, y que cuenta con un reducido y poco preparado personal para hacer frente a tareas demandantes.

Un funcionalismo que se asume como esquema de observación transversal a la sociedad puede ser una herramienta útil para las ciencias sociales contemporáneas, en tanto se mantenga abierta su forma a la inestabilidad, es decir, a poner en tela de juicio la propia forma, a preguntarse por la función del funcionalismo.

A pesar de todo embate, el hecho que el funcionalismo no haya desaparecido del todo del panorama teórico de las ciencias sociales, puede ser sintomático de que su longevidad, aunque achacosa, se deba a que no se conocen todavía otras soluciones a sus problemas más profundos. A diferencia de corrientes teóricas que gozaron de una vigencia más bien fugaz -como el pomposo evolucionismo antropológico o las fallidas teorías posmodernas- el funcionalismo se encuentra, para bien o para mal, estable dentro de su gravedad.

Hugo Cadenas - Doctor en Sociología, Universidad Ludwig Maximilian de Munich, Alemania. Profesor Asistente, Departamento de Antropología. Miembro del Comité Académico del Magíster en Análisis Sistémico Aplicado a la Sociedad. $>$ cadenas@u.uchile.cl

\section{Referencias}

1. ALEXANDER, J.C. Twenty Lectures. Sociological Theory since World War II. New York: Columbia University Press, 1987.

2. ALEXANDER, J.C.; COLOMY, P. El neofuncionalismo hoy - reconstruyendo una tradición teórica. Sociológica, 7 (20), 1992.

3. ARCHER, M. Culture and Agency. The Place of Culture in Social Theory. New York: Cambridge University Press. 1996.

4. BLUMER, H. Social Psychology. In: SCHMIDT, E.P (Ed) Man and Society: a substantive introduction to the social science. New York: Prentice-Hall, Inc, 1937. pp. 144-198. 
5. BLUMER, H. Symbolic Interactionism: Perspective and Method. London: University of California Press, 1969.

6. CADENAS, H. Organizaciones y grupos: loose coupling/strict coupling. In: ARNOLD, M.; CADENAS, H.; URQUIZA, A. (Eds.), La organización de las organizaciones sociales: Aplicaciones desde perspectivas sistémicas. Santiago de Chile: RIL Editores, 2014, pp. 153-170.

7. COMTE, A. Principios de filosofía positiva. Santiago de Chile: Imprenta de la Librería del Mercurio, 1875.

8. DURKHEIM, E. Las reglas del método sociológico. México DF: FCE, 2001.

9. DURKHEIM, E. La división del trabajo social. México DF: Colofón. [1893], 2007.

10. GARFINKEL, H. Studies in Ethnomethodology. Englewood Cliffs, NJ: Prentice-Hall, 1967.

11. HABERMAS, J.; LUHMANN, N. Theorie der Gesellschaft oder Sozialtechnologie - Was leistet die Systemforschung? Frankfurt aM: Suhrkamp, 1971.

12. HORKHEIMER, M.; ADORNO, T. W. Dialéctica de la Ilustración. Fragmentos filosóficos. Madrid: Trotta, 1998.

13. LATOUR, B. The Pasteurization of France. Cambridge, Mass: Harvard University Press, 1993.

14. LUHMANN, N. Funktion und Kausalität. In: LUHMANN, N. Soziologische Aufklärung 1: Aufsätze zur Theorie sozialer Systeme, Opladen: Westdeutscher Verlag, 1970, pp. 9-30.

15. LUHMANN, N. Interaktion, Organisation, Gesellschaft. Anwendungen der Systemtheorie. In: LUHMANN, N. Soziologische Aufklärung 2: Aufsätze zur Theorie der Gesellschaft, Opladen: Westdeutscher Verlag, 1975, pp. 9-20.

16. LUHMANN, N. Die Gesellschaft der Gesellschaft. Frankfurt a.M.: Suhrkamp, 1997.

17. MALINOWSKI, B. The Group and the Individual in Functional Analysis. The American Journal of Sociology, 44(6), 1939, pp. 938-964.

18. MALINOWSKI, B. A Scientific Theory of Culture. And Other Essays. New York: Oxford University Press, 1960.

19. MERTON R. K. Social Theory and Social Structure. New York: Free Press, 1949.

20. MÜNCH, R. Theorie des Handelns. Zur Rekonstruktion der Beiträge von Talcott Parsons, Emile Durkheim und Max Weber. Frankfurt aM: Suhrkamp, 1982.

21. NASSEHI, A. Rethinking Functionalism. In: NASSEHI, A. Gesellschaft der Gegenwarten. Studien zur Theorie der modernen Gesellschaft II. Frankfurt aM: Suhrkamp, 2011, pp. 45-69. 
22. PARSONS, T. The Structure of Social Action. New York: The Free Press, 1937.

23. PARSONS, T. The Social System. New York: The Free Press, 1951.

24. PARSONS, T. Malinowski and the Theory of Social Systems. In: FIRTH, R. (Ed.) Man and Culture: an Evaluation of the Work of Bronislaw Malinowski. London: Routledge \& Kegan Paul, 1960, pp. 53-70.

25. PARSONS, T. On the Concept of Influence. The Public Opinion Quarterly, 27(1), 1963a, pp. 37-62.

26. PARSONS, T. On the Concept of Political Power. Proceedings of the American Philosophical Society, 107(3), 1963b, pp. 232-262.

27. PARSONS, T. Evolutionary Universals in Society. American Sociological Review, 29(3), 1964, pp. 339-357.

28. PARSONS, T. Societies: Evolutionary and Comparative Perspectives. Englewood Cliffs, N.J.: Prentice-Hall, 1966.

29. PARSONS, T. On the Concept of Value-Commitments. Sociological Inquiry, 38(2), 1968, pp. 135-160.

30. PARSONS, T. On Building Social System Theory: A Personal History. Daedalus, 99(4), 1970, pp. 826-881.

31. PARSONS, T. The System of Modern Societies. Englewood Cliffs, N.J.: Prentice-Hall, 1971.

32. PARSONS, T.; SHILS, E. (Eds.). Toward a General Theory of Action. Cambridge, Mass: Harvard University Press, 1951.

33. PARSONS, T.; BALES, R.; SHILS E. Working Papers in the Theory of Action. New York: The Free Press, 1953.

34. PARSONS, T.; SMELSER, N. J. Economy and Society: A Study in the Integration of Economic and Social Theory. London: Routledge \& Kegan Paul, 1956.

35. RADCLIFFE-BROWN, A. R. Structure and Function in Primitive Society. Glencoe, III: The Free Press, 1952.

36. VERHOEVEN, J. An Interview with Erving Goffman, 1980. Research on Language and Social Interaction, 26(3), 1993, pp. 317-348.

Recebido em: 10/12/2015

Aceite final em: 22/02/2016 